\title{
Efecto de la Adición de Jarabes de Sacarosa y Fructosa en el Comportamiento Reológico de Miel de Abejas
}

\author{
Ricardo D. Andrade ${ }^{(1,2) \star}$, Ramiro Torres ${ }^{(1)}$, Ana M. Pérez ${ }^{(3)}$ \\ (1) Grupo de Investigación GIPPAL, Facultad de Ingeniería, Universidad de Córdoba, Km 12 Vía Cereté- \\ Ciénaga de Oro, Colombia (rdandrade@correo.unicordoba.edu.co). \\ (2) Grupo Investigaciones en Procesos Agroindustriales, Facultad de Ingeniería, Universidad de Córdoba, \\ Km 12 Vía Cereté-Ciénaga de Oro, Colombia \\ (3) Maestría en Ciencias Agroalimentarias, Universidad de Córdoba, Colombia.
}

${ }^{*}$ Autor a quien debe ir dirigida la correspondencia.

Recibido Jun. 22, 2015; Aceptado Ago. 21, 2015; Versión final Sep. 29, 2015, Publicado Feb. 2016

\section{Resumen}

Se evaluó el efecto de la adición de jarabes de sacarosa y fructosa en las propiedades reológicas de la miel de abejas producida en dos zonas, Sabanas y Montes de María, del departamento de Sucre en Colombia. A las mieles puras se les adicionó jarabe de sacarosa o fructosa en porcentajes de $0,10,20$, 30,40 y $50 \%$. Las propiedades reológicas se determinaron utilizando un reómetro AR1500EX TA Instruments, plato de $40 \mathrm{~mm}$, abertura (gap) de $1500 \mu \mathrm{m}$ y temperatura de $30^{\circ} \mathrm{C}$, con gradiente de cizalla

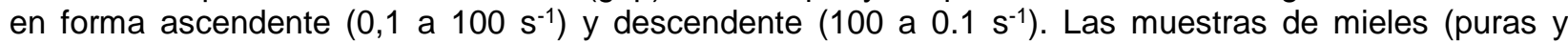
adulteradas) presentan un comportamiento newtoniano. Además, el aumento en la cantidad de los jarabes adicionados disminuye la viscosidad de las mieles. Por lo tanto el valor de la viscosidad puede utilizarse para determinar la adulteración de mieles por adición de jarabes de sacarosa y fructosa.

\section{Effect of the Addition of Saccharose and Fructose Syrup on the Rheological Behavior of Honey Bees}

\begin{abstract}
The effect of the addition of saccharose and fructose syrups on the rheological properties of honey produced in two regions, Sabanas and Montes de María, in the Department of Sucre in Colombia was determined. Sucrose or fructose syrup in percentages of $0,10,20,30,40$ and $50 \%$ were added to pure honey. The rheological properties were determined using a TA Instruments rheometer AR1500EX, plate of $40 \mathrm{~mm}$, gap of $1500 \mu \mathrm{m}$ and at temperature of $30^{\circ} \mathrm{C}$. The shear rate was increased and decreased between 0.1 and $100 \mathrm{~s}^{-1}$. Samples of honey (pure and adulterated) have newtonian behavior. Furthermore, the increased in the amount of syrups makes the viscosity of honey to decrease. Thus, viscosity can be used for determining adulteration of honey by addition of fructose or saccharose syrups.
\end{abstract}

Keywords: honey bee; rheological properties; newtonian behavior; adulteration 


\section{INTRODUCCIÓN}

La miel es una sustancia dulce y natural, producida por abejas Apis mallifera o diferentes subespecies, a partir del néctar de las plantas o de otras secreciones extra florales. los componentes más comunes que se encuentran en la miel son el agua $(17,1 \%)$, azúcares $(82,4 \%)$, proteínas $(0,1 \%)$ y otros componentes que incluyen vitaminas, minerales, sustancias aromáticas y ácidos orgánicos, entre otros $(0,4 \%)$ (Bogdanov, 2003). Está compuesta esencialmente de diferentes azúcares, sobre todo fructosa y glucosa, así como otras sustancias tales como ácidos orgánicos, enzimas y partículas sólidas provenientes de su recolección (Moguel et al., 2005). Aunque la fructosa y glucosa son los monosacáridos predominantes (entre 60 y $85 \%$ ), también contiene maltosa y sacarosa. La relación de fructosa-glucosa es un factor determinante de la velocidad de cristalización de la miel, y además es responsable en buena parte de sus propiedades reológicas (Witczak et al., 2011).

Hay también otros factores, tales como otros hidratos de carbono, los granos de polen, burbujas de aire y partículas pequeñas que pueden actuar como cristales de siembra y contribuir a la cristalización de la miel (Turhan et al., 2008). Las caracteristicas organolépticas (color, aroma, sabor), fisicoquímicas y nutricionales de la miel varían con la fuente de néctar o de mielato, cantidad de recurso recolectado por las abejas de estas fuentes, origen geografico y tecnología del proceso productivo (Nantes-Parra et al., 2013). Por lo tanto, la clasificación geográfica y botánica han tomado gran importancia como factores de diferenciación de las mieles para su comercialización (Suescún y Vit, 2008) y en consecuencia ahora los productores deben preocuparse por su caracterización.

En Colombia, la cadena apícola incluye la obtención de miel, polen, propóleos, cera, jalea y larvas; pero la mayor parte de la actividad, se ha concentrado en la producción de la miel, la cual, aún no se ha considerado como competitiva para el desarrollo agropecuario del país y la producción nacional no está suficientemente consolidada como para aspirar a posicionarse en el mercado internacional. No obstante, la variada vegetación de las regiones apicultoras Colombianas le permite tener cualidades específicas que la clasifican entre las mejores del mundo (Agrocadenas, 2006). Entre estas regiones, la costa Caribe Colombiana tiene gran potencial para la obtención de miel, específicamente aquellas zonas que aún albergan ecosistemas de bosque natural y donde las condiciones climáticas de luz, temperatura y humedad favorecen la floración de la vegetación. Los departamentos apícolas más evolucionados son Bolívar, Sucre, Magdalena y Atlántico, con rendimientos hasta de $40 \mathrm{Kg} /$ colmena al año, una cifra bastante prometedora, si se compara con el departamento de Norte de Santander (30 Kg/colmena), y en el resto del país la producción llega a $20 \mathrm{Kg} /$ colmena en promedio anual (Laverde et al., 2010).

Debido al alto valor nutritivo y características del sabor de la miel, esta presenta un precio mucho mayor que otros endulzantes o edulcorantes; por lo cual se pueden presentar adulteraciones con adición de sustancias como sacarosa, fructosa y otro tipo de azúcar, con el fin de disminuir el costo de la miel (Sivakeseva y Irudayaraj, 2002; Guler et al., 2007). Para detectar estas adulteraciones en la miel se han utilizado diferentes métodos, entre los que se pueden citar: análisis electroquímico, enzimáticos, cromatografía, resonancia magnética nuclear, entre otros. Sin embargo, la mayoría de estos métodos necesitan procedimientos largos, o equipos especializados. Las propiedades reológicas de la miel de abejas tienen gran importancia, debido a que es posible una diferenciación, clasificación e identificación de acuerdo a sus diferentes orígenes botánicos, biogeográficos y entomológicos mediante un análisis reológico y el establecimiento de los parámetros característicos de flujo (Witczak et al., 2011; Dobre et al., 2012). Además, en los últimos años se han realizado estudios que demuestran la importancia de las características reológicas como herramienta para identificar la adulteración de mieles, por ejemplo: Yilmaz et al. (2014) comprobaron el cambio de comportamiento de flujo (disminución de la viscosidad), viscoelasticidad y esfuerzo de fluencia de mieles de Turquía adulteradas con diferentes proporciones de jarabe de sacarosa y fructosa.

Por lo anterior, el presente trabajo tiene por objetivo determinar la influencia de la adición de jarabes de sacarosa y fructosa (adulteración) en las propiedades reológicas de miel de abejas (Apis mellifera) producida en dos zonas geográficas diferentes del departamento de Sucre: Sabanas (Sampúes) y Montes de María (El Piñal). Cabe destacar que la subregión Sabanas está situada en la parte central de departamento de Sucre, y la conforman numerosas sierras y colinas formando ondulaciones que van desde los 70 hasta $185 \mathrm{msnm}$. Por otra parte, la subregión Montes de María está ubicada en la parte nororiental del departamento Sucre, corresponde a una zona de bosque seco tropical y su paisaje característico es la montaña. 


\section{MATERIALES Y MÉTODOS}

\section{Materia Prima:}

La miel de abejas producida por abejas del genero Apis mellifera, se obtuvo de mercados locales en dos zonas geográficas diferentes del departamento de Sucre: Sabanas (Sampúes) y Montes de María (El Piñal). Las muestras fueron almacenadas en refrigeración, hasta la realización de las pruebas reológicas.

Preparación de jarabes de sacarosa y fructosa y muestras de miel adulteradas.

Los jarabes de sacarosa y fructosa, se prepararon añadiendo lentamente $450 \mathrm{~g}$ de sacarosa o fructosa en polvo a $300 \mathrm{~g}$ de agua destilada, seguido por una mezcla con agitador. Ambos jarabes se concentraron hasta aproximadamente $64 \stackrel{\circ}{\circ}$ Brix mediante calentamiento directo en una plancha eléctrica. Para la preparación de muestras de miel adulteradas, se adicionaron el jarabe de sacarosa o fructosa a las muestras de miel naturales en porcentajes de $0,10,20,30,40$ y $50 \%$, w/w.

\section{Determinación de parámetros reológicos:}

Se tomaron $10 \mathrm{~mL}$ de muestra de miel y se determinaron las propiedades reológicas en un reómetro AR1500EX TA Instruments, con un accesorio de plato de $40 \mathrm{~mm}$, un gap de $1500 \mu \mathrm{m}$ y una temperatura de $30^{\circ} \mathrm{C}$, variando el gradiente de cizalla en una rampa continua de 0,1 a $100 \mathrm{~s}^{-1}$ en forma creciente y luego en forma decreciente de 100 a $0,1 \mathrm{~s}^{-1}$, para las diferentes concentraciones de jarabe adicionado a las mieles de las dos localidades. El equipo se controló mediante el software Rheology Advantage Instrument Control AR y los datos se adquirieron y procesaron mediante TA Análisis de datos v.2.0 y Microsoft Excel 2010.

\section{Diseño experimental y análisis estadístico:}

El experimento se condujo bajo un diseño factorial $2 \times 2 \times 5$, con los factores: zona de procedencia de la miel (Sabanas y Montes de maría), tipo de adulterante (jarabe de sacarosa y fructosa), y el porcentaje de adición de jarabe $(10,20,30,40,50 \% \mathrm{w} / \mathrm{w})$, cada tratamiento con dos repeticiones, para un total de 60 unidades experimentales, con la variable respuesta viscosidad. Se realizó un análisis de varianza para determinar si existía efecto significativo de los factores sobre la variable de repuesta. Para determinar si las mieles puras (control) presentavan diferencias significativas con las mieles adulteradas (adicionadas con los distintos jarabes) se realizó una prueba de Dunnett's a un nivel de significancia del $5 \%$, utilizando el software JMP 9.01 (SAS Institute).

\section{RESULTADOS Y ANÁLISIS}

En la figura 1 se presentan los reogramas de mezcla de mieles con un $20 \%$ de jarabe de sacarosa procedentes de la región de Sabana y Montes de María. Se observa que no hay diferencia entre las curvas de ascenso y descenso para las mieles, este comportamiento lo presentaron para las mieles puras y adulteradas, independiente de la zona de procedencia. Para corroborar este comportamiento, se realizó un análisis de comparación de muestras pareadas (ascendente y descendente). De acuerdo a la prueba de tStudent, no hubo diferencias significativas $(p=0,377)$ entre los valores obtenidos de forma ascendente y descendente. Por lo cual se puede afirmar que las mieles puras y adulteradas con jarabe de sacarosa y fructosa, presentan un comportamiento independiente del tiempo.

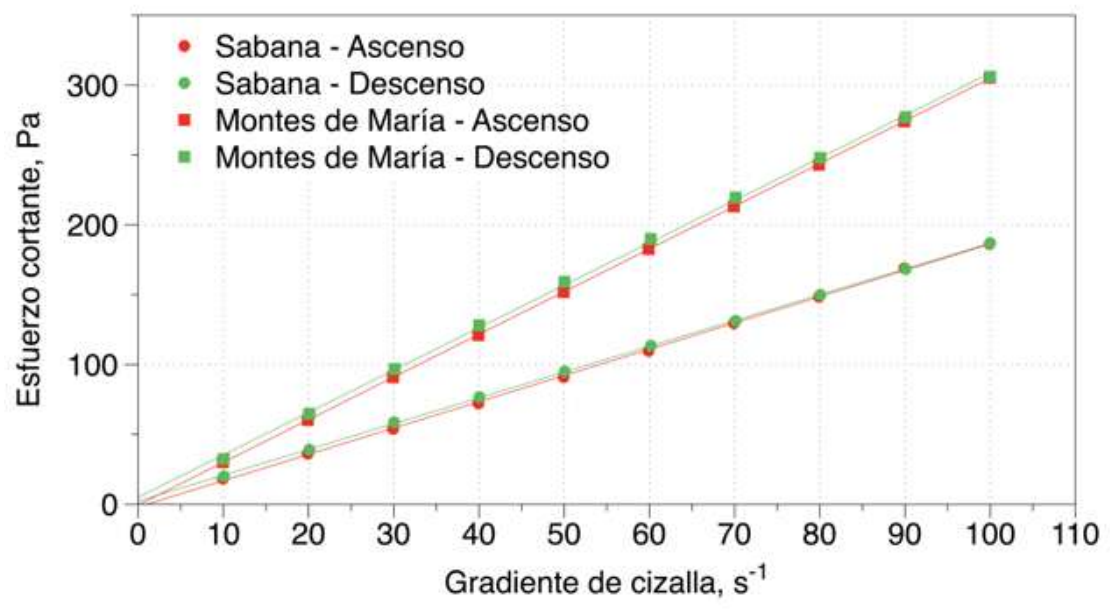

Fig. 1: Reogramas de ascenso y descenso de mieles provenientes de la región de Sabanas y Montes de María 
En la figura 2 se presentan los reogramas característicos de las mieles estudiadas. Todas las muestras se ajustaron a una línea recta $\left(R^{2}>0,99\right.$ y $\left.E S<0,0022\right)$ y pasa por el origen, por lo cual se puede afirmar que su comportamiento es de un fluido Newtoniano. Este comportamiento ha sido reportado por varios autores para mieles de diferentes regiones, tales como en Jordania (Abu-Jdayil et al., 2002); Grecia (Lazaridou et al., 2004) y Rumania (Dobre et al., 2012). Contrario a estos resultados, algunas mieles han sido reportadas con un comportamiento no Newtoniano, como mieles provenientes del estado de Piaú, Brasil (Queiroz et al., 2007), de la India (Ahmed et al., 2007) y Polonia (Witczak, et al., 2011).

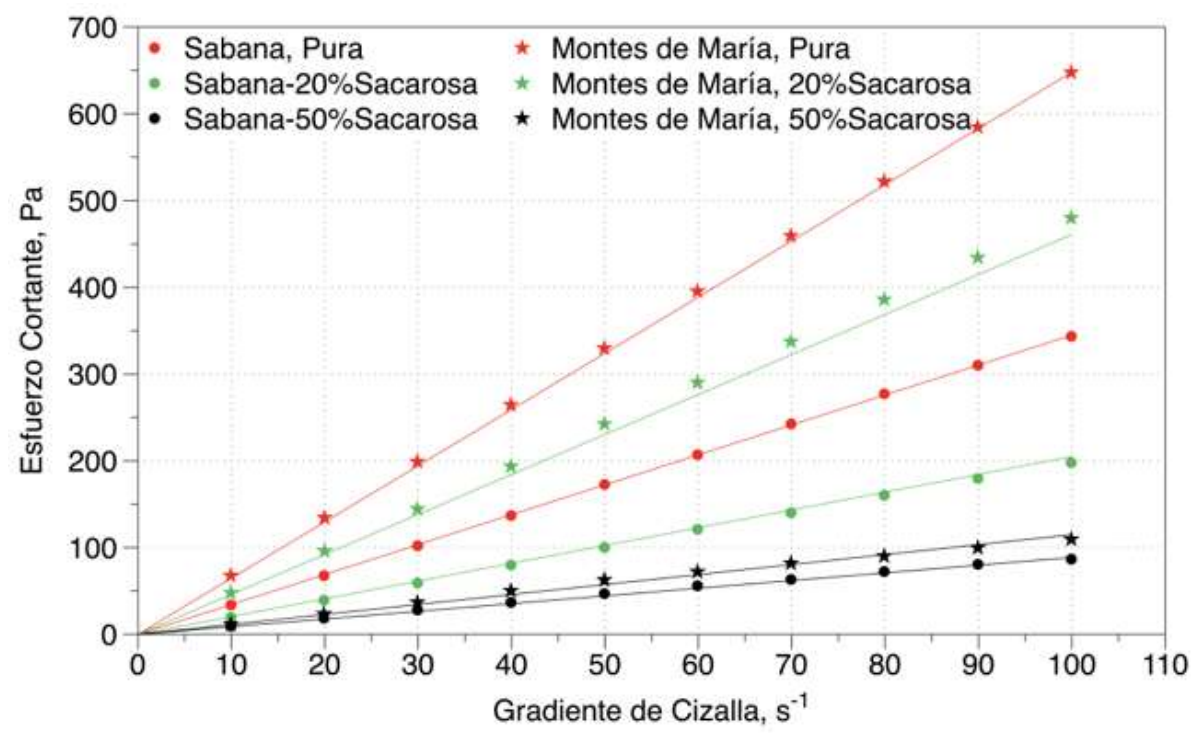

Fig. 2: Reogramas de mieles provenientes de la región de Sabanas y Montes de María (Sucre, Colombia)

El análisis de varianza (ANOVA) muestra que la procedencia de la miel, el tipo de adulterante y el porcentaje de adición de jarabe tuvieron un efecto significativo (a un nivel de significancia del $5 \%$ ) sobre la variable de repuesta viscosidad. La miel pura procedente de la región de Sabanas (Sucre) presenta una viscosidad de $3449 \pm 43 \mathrm{mPa}$.s mientras que la procedente de Los Montes de María (Sucre) tiene una viscosidad de $6480 \pm 66 \mathrm{mPa}$.s. Este ultimo valor es similar a los reportados para mieles procedentes de Turquía, $6531 \mathrm{mPa}$.s (Yilmaz et al., 2014). La viscosidad de las mieles se ven afectadas por la procedencia de está (Sabanas y Montes de María), debido a que las características y las propiedades fisicoquímicas de la miel varían con las fuentes florales utilizadas por las abejas, las condiciones climáticas y geográficas (Nantes-Parra et al., 2013).

En la Tabla 1 se muestra la viscosidad de las mieles puras y adulteradas con adición de jarabe de sacarosa y fructosa. Se observa que la adición de adulterante afecta la viscosidad de las mieles. Para determinar si existía diferencia significativa entre las mieles adulteradas (adición de jarabe de sacarosa y fructosa) y las mieles puras (control), se realizó la prueba de comparación de Dunnett's. La cual arrojó que todas las mieles adulteradas presentaban diferencia significativa $(p<0,05)$ con las mieles puras, por lo cual se puede usar la viscosidad como un parámetro para determinar la adulteración de mieles adicionadas con un mínimo de $10 \%$ de sacarosa o fructosa.

Tabla 1: Viscosidad de las mieles puras y adulteradas con jarabe de sacarosa y fructosa

\begin{tabular}{|c|c|c|c|c|}
\hline \multirow{2}{*}{ Adulterantes, \% } & \multicolumn{2}{|c|}{$\begin{array}{c}\text { Viscosidad de la Miel de Sabanas, } \\
\text { mPa.s }\end{array}$} & Viscosidad de la Miel de Los Montes de María, \\
mPa.s
\end{tabular}


En la Fig. 3 se muestra el efecto de la adición del adulterante (jarabe de sacarosa y fructosa) sobre la viscosidad de las mieles. Se observa que a medida que aumenta la cantidad de adulterante adicionado ocasiona una disminución de la viscosidad, independientemente del tipo de jarabe utilizado. Este resultado se puede explicar por la viscosidad de los jarabes de sacarosa (300 cP) y fructosa (1265 mPa.s), que son menores que la de las mieles utilizadas. Sin embargo, el comportamiento no es el mismo. En la Tabla 2 se muestra que la adición de jarabe de sacarosa en las distintas mieles se ajustaron a una línea recta $\left(\mathrm{R}^{2} \geq\right.$ $0,95)$, mientras que la adición de jarabe de fructosa en las distintas mieles se ajuntaron mejor a una ecuación exponencial $\left(R^{2} \geq 0,92\right)$.

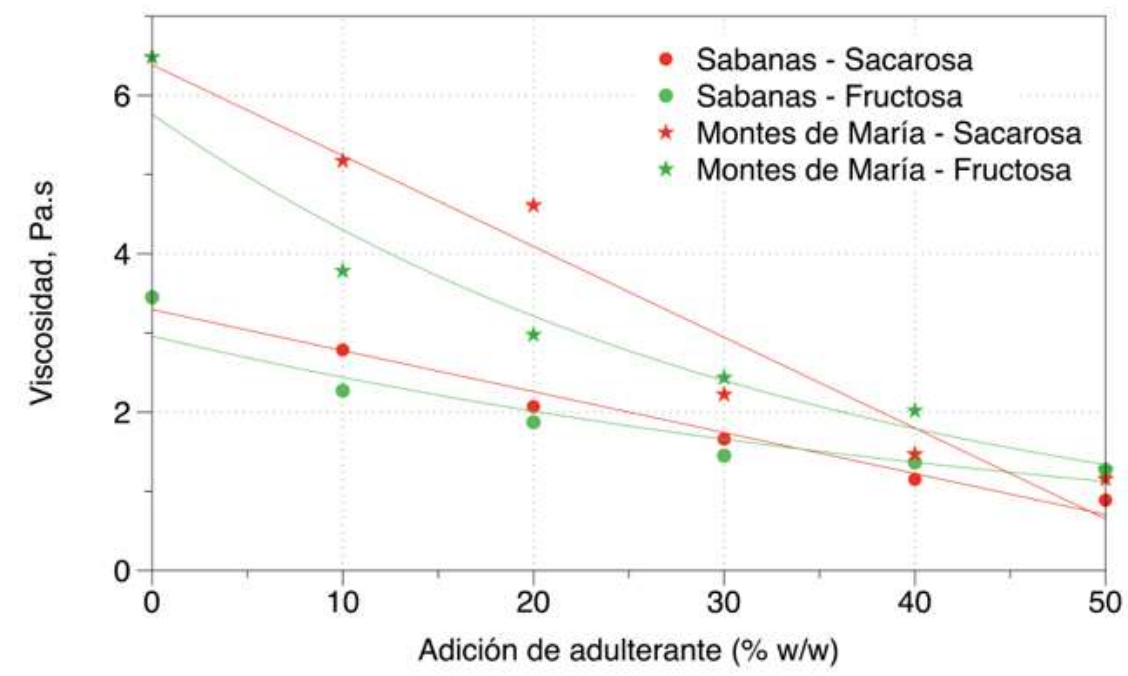

Fig. 3: Efecto de la adición de adulterante en la viscosidad de las mieles

Tabla 2: Modelos de ajuste de mieles adulteradas con Sacarosa (S) y Fructosa (F)

\begin{tabular}{|l|l|l|c|}
\hline Tipo de miel & Adulterantes & Modelo & $\mathrm{R}^{2}$ \\
\hline \multirow{2}{*}{ Región de Sabanas } & Sacarosa & $\mu=3,295-0,0518 \mathrm{~S}$ & 0,978 \\
\cline { 2 - 4 } & Fructosa & $\mu=2,959 \exp (-0,0193 \mathrm{~F})$ & 0,928 \\
\hline \multirow{2}{*}{ Región Montes de María } & Sacarosa & $\mu=6,386-0,0115 \mathrm{~S}$ & 0,952 \\
\cline { 2 - 4 } & Fructosa & $\mu=5,791 \exp (-0,0292 \mathrm{~F})$ & 0,966 \\
\hline
\end{tabular}

A pesar que el jarabe de fructosa tiene una viscosidad mayor que la del jarabe de sacarosa, se observa que la adición de jarabe de fructosa ocasiona una disminución mayor que la adición de jarabe de sacarosa, este comportamiento es principalmente a porcentajes menores del 10\% de adulterante. Por ejemplo, una adición de $10 \%$ de jarabe de fructosa a las mieles provenientes de la región de Sabanas y Montes de María (Sucre) ocasiona una disminución de la viscosidad de aproximadamente un $38 \%$, mientras que la adición de $10 \%$ de jarabe de sacarosa a estas mieles solo ocasiona una disminución de $20 \%$. Esto se puede deber a que la adición del jarabe de sacarosa cambia en mayor grado la relación fructosa-glucosa, que es un parámetro que afecta la viscosidad en las mieles. Por otra parte, estos resultados concuerdan con los reportados por Yilmaz et al. (2014), que para mieles provenientes de Turquía, la adición de jarabe de fructosa y sacarosa provocaron una disminución del 38 y $13,5 \%$, respectivamente.

\section{CONCLUSIONES}

Las mieles de abejas procedentes de las zonas geográficas de Sabanas y los Montes de María (Sucre, Colombia) presentan un comportamiento Newtoniano. El incremento de la adición de jarabe de sacarosa o fructosa disminuye la viscosidad de las mieles. La adulteración de las mieles por la adición de jarabe de sacarosa o fructosa puede detectarse por la medida de la viscosidad, y debido a que son un fluido Newtoniano, está prueba se puede realizar con un viscosímetro sencillo (Capilar, caída de bola).

\section{REFERENCIAS}

Abu-Jdayil, B., A. Ghzawi, K. Al-Malah y S. Zaitoun. Heat effect on rheology of light- and dark-colored honey, Journal of Food Engineering, 51 (1), 33-38 (2002) 
Agrocadenas. La cadena de las abejas y la apicultura en Colombia (en linea), 2006. https://bancayagro.files.wordpress.com/2008/06/caracterizacion_abejas2.pdf. Acceso: 8 de Junio (2015)

Ahmed, J., S.T. Prabhu, G.S.V. Raghavan y M. Ngadi. Physico-chemical, rheological, calorimetric and dielectric behavior of selected Indian honey. Journal of Food Engineering, 79 (4), 1207-1213 (2007)

Bogdanov, S. Current status of analytical methods for the detection of residues in bee products. Apiacta, 38 , 190-193 (2003)

Dobre, I., L. Georgescu, P. Alexe, O. Escuredo y M.C. Seijo. Rheological behavior of different honey types from Romania. Food Research International, 49(1), 126-132 (2012)

Guler, A., A. Bakan, C. Nisbet y O. Yavuz. Determination of important biochemical properties of honey to discriminate pure and adulterated honey with sucrose (Saccharum officinarum L.) syrup. Food Chemistry, 105(3), 1119-1125 (2007)

Laverde, J. L. Egea, D. Rodríguez, y J. Peña. Agenda prospectiva de investigación y desarrollo tecnológico para la cadena productiva de las abejas y la apicultura en Colombia con énfasis en miel de abejas (en linea), 2010. http://digitool.gsl.com.mx:1801/webclient/StreamGate?folder_id=0\&dvs=1434060158864 894. Acceso: 8 de Junio (2015)

Lazaridou, A., C.G. Biliaderis, N. Bacandritsos, y A.G. Sabatini. Composition, thermal and rheological behaviour of selected Greek honeys. Journal of Food Engineering, 64(1), 9-21(2004)

Moguel, Y.B., C. Echazarret y R. Mora. Calidad fisicoquímica de la miel de abeja Apis mellifera producida en el estado de Yucatán durante diferentes etapas del proceso de producción y tipos de floración. Revista Mexicana de ciencias pecuarias, 43(3), 323-334 (2005)

Nates-Parra G., P.M. Montoya, F.J. Chamorro, N. Ramírez, C. Giraldo y D. Obregón. Origen geográfico y botánico de mieles de Apis Mellifera (apidae) en cuatro departamentos de Colombia. Acta Biológica Colombiana, 18(3), 427-438 (2013)

Queiroz, A.J., R.M.F. Figueirêdo, C.L. da Silva y M.E. Mata. Comportamento reológico de méis de florada de silvestre. Revista Brasileira de Engenharia Agrícola e Ambiental, 11(2), 190-194 (2007)

Sivakeseva, S. y J. Irudayaraj. Classification of simple and complex sugar adulterants in honey by midinfrared spectroscopy. International Journal of Food Science and Technology, 37(4), 351-360 (2002)

Suescún L. y P. Vit P. Control de calidad de la miel de abejas producida como propuesta para un proyecto de servicio comunitario obligatorio. Fuerza Farmacéutica, 12(1), 6-15 (2008)

Turhan, I., N. Tetik, M. Karha, F. Gurel y H.R. Tavukcuoglu. Quality of honeys influenced by thermal treatment. LWT-Food Science and Technology, 41 (8), 1396-1399 (2008)

Witczak, M., L. Juszczak y D. Gałkowska. Non-Newtonian behaviour of heather honey. Journal of Food Engineering, 104(4), 532-537 (2011)

Yilmaz, M.T., N.B. Tatlisu, O.S. Toker, S. Karaman, E. Dertli, O. Sagdic y M. Arici. Steady, dynamic and creep rheological analysis as a novel approach to detect honey adulteration by fructose and saccharose syrups: Correlations with HPLC-RID results. Food Research International, 64, 634-646 (2014) 